\title{
Global Conservative Solutions of a Generalized Two-Component Camassa-Holm System
}

\author{
Feng Zhang, ${ }^{1}$ Han Yang, ${ }^{1}$ and Yonghong $\mathrm{Wu}^{2}$ \\ ${ }^{1}$ School of Mathematics, Southwest Jiaotong University, Chengdu 610031, China \\ ${ }^{2}$ Department of Mathematics and Statistics, Curtin University, Perth, WA 6845, Australia
}

Correspondence should be addressed to Han Yang; hanyang95@263.net

Received 2 December 2013; Accepted 23 December 2013; Published 20 February 2014

Academic Editor: Guangchen Wang

Copyright (c) 2014 Feng Zhang et al. This is an open access article distributed under the Creative Commons Attribution License, which permits unrestricted use, distribution, and reproduction in any medium, provided the original work is properly cited.

The Cauchy problem for a generalized two-component Camassa-Holm system is investigated. Following the idea of fixed points and using new sets of independent and dependent variables, the existence of the global conservative solutions for the system is established.

\section{Introduction}

We consider a generalized two-component Camassa-Holm system

$$
\begin{gathered}
u_{t}-u_{t x x}+[g(u)]_{x}=2 u_{x} u_{x x}+u u_{x x x}-\eta \bar{\eta}_{x}, \\
t>0, x \in R, \\
\eta_{t}+(u \eta)_{x}=0, \quad t>0, x \in R, \\
u(0, x)=u_{0}(x), \quad \eta(0, x)=\eta_{0}(x), \quad x \in R,
\end{gathered}
$$

which is a model for wave motion on shallow water; $u(t, x)$ describes the horizontal velocity of the fluid, $g(u) \in C^{\infty}(R)$, $g(0)=0, \eta$ is horizontal deviation of the free surface elevation from equilibrium (or depth, in the shallow-water interpretation), and $\eta=\left(1-\partial_{x}^{2}\right) \bar{\eta}$, where $\bar{\eta}$ indicates locally averaged density. The classical Camassa-Holm equation in [1] has the form

$$
u_{t}-u_{t x x}+2 k u_{x}+3 u u_{x}=2 u_{x} u_{x x}+u u_{x x x} .
$$

Combining its integrability property with free surface elevation dynamics in [2], the authors have extended the Camassa-Holm equation to two-component Camassa-Holm system $(\mathrm{CH} 2)$ :

$$
\begin{gathered}
u_{t}-u_{t x x}+3 u u_{x}=2 u_{x} u_{x x}+u u_{x x x}-\eta \eta_{x} \\
\eta_{t}+(u \eta)_{x}=0
\end{gathered}
$$

Moreover, the local well-posedness and blow-up solutions for $\mathrm{CH} 2$ have been established in $[3,4]$. It was shown that the system has strong solutions which blow up in finite time [5]. Using the localization analysis in the transport equation, Gui and Liu [6] obtained the global solutions of (3) in the Sobolev space $H^{s} \times H^{s-1}$ with $s>3 / 2$. Hu [7] considered the weakly dissipative $\mathrm{CH} 2$ which includes a nonlinearly dissipative term $L(u)$, where $L$ is a differential operator or a quasidifferential operator. It has been shown that $\mathrm{CH} 2$ system possesses peakon solutions in $u$ and cornerlike solutions in $\eta$, but singular solutions do not exist for its density variable [8]. By setting $\eta=\left(1-\partial_{x}^{2}\right) \bar{\eta}$, Holm et al. [8] derived the modified $\mathrm{CH} 2(\mathrm{MCH} 2)$ :

$$
\begin{gathered}
u_{t}-u_{t x x}+3 u u_{x}=2 u_{x} u_{x x}+u u_{x x x}-\eta \bar{\eta}_{x} \\
\eta_{t}+(u \eta)_{x}=0
\end{gathered}
$$

where the $\bar{\eta}$ is averaged or filtered density. Then, this slight modification of the system $\mathrm{CH} 2$ leads to the system $\mathrm{MCH} 2$. In [9], the global conservative and dissipative solutions of (4) have been testified.

The Cauchy problem for (2) has been studied extensively. It has been shown that this equation has global strong solutions [10-12]. On the other hand, it has global weak solutions in $H^{1}(R)$ [13-15]. Weak solutions for a weakly dissipative Camassa-Holm equation have been obtained in [16]. Moreover, the global and dissipative solutions have been 
established in [17-20]. It is worth remarking that Bressan and Constantin [17] derived the existence of global conservative solutions to (2). The essential point in [17] is to introduce some independent and dependent variables.

For $g(u)=a u^{m}+k u(a, k$ is constant and $m$ is positive integer), the generalized Camassa-Holm equation

$$
\begin{gathered}
u_{t}-u_{t x x}+[g(u)]_{x}=2 u_{x} u_{x x}+u u_{x x x}, \quad t>0, x \in R, \\
u(0, x)=u_{0}(x), \quad x \in R
\end{gathered}
$$

has been discussed in [21, 22], and the existence of travelling waves solutions and peaked solitary wave solutions has been found. Mustafa [23] and Yin [24] obtained results of local well-posedness. The global conservative solutions and dissipative solutions of (5) have been established in [25]. However, the conservative solutions for the problem (1) have not been discussed yet. The aim of this paper is to establish the existence of global conservations for the problem (1) and prove uniqueness and continuous dependence on the initial data.

This paper is organized as follows. The preliminary is given in Section 2. In Section 3, we come up with an equivalent semilinear system for the problem (1) and the global solution of this system is constructed. In Section 4, we prove that the semilinear system yields global conservation solutions to the problem (1).

\section{Preliminaries}

2.1. The Basic Equations. Firstly, we give some notations.

We define Banach space $X$ with the norm $\|\cdot\|_{X}$ and let $p(x)=(1 / 2) e^{-|x|}, x \in R$; then $\left(1-\partial_{x}^{2}\right)^{-1} f=p * f$ for all $f \in L^{2}$. We set $\varphi=\bar{\eta}-\bar{\eta}_{0}\left(\bar{\eta}_{0}\right.$ is a constant $)$ and $\eta=\varphi-\varphi_{x x}$; then $\varphi_{x}=\bar{\eta}_{x}, p * \eta=\varphi$.

We rewrite the equivalent form of (1) as follows:

$$
\begin{gathered}
u_{t}+u u_{x}=-\Psi_{1 x}, \quad t>0, x \in R, \\
\varphi_{t}+u \varphi_{x}=-\Psi_{2 x}-\Psi_{3}, \quad t>0, \quad x \in R, \\
u(0, x)=u_{0}(x), \quad \varphi(0, x)=\varphi_{0}(x), \quad x \in R,
\end{gathered}
$$

with initial data $\left(u_{0}(x), \varphi_{0}(x)\right) \in H^{1} \times\left(H^{1} \cap W^{1, \infty}\right)$ and in which $\Psi_{i}(i=1,2,3)$ are defined by

$$
\begin{gathered}
\Psi_{1}=p *\left(u^{2}+\frac{1}{2} u_{x}^{2}+g(u)+\frac{1}{2} \varphi^{2}-\frac{1}{2} \varphi_{x}^{2}\right), \\
\Psi_{2}=p *\left(u_{x} \varphi_{x}\right), \\
\Psi_{3}=p *\left(u_{x} \varphi\right),
\end{gathered}
$$

where $p(x)=(1 / 2) e^{-|x|}, g(u) \in C^{\infty}(R)$, and $g(0)=0$. If $u$ is in $H^{1}(R)$, due to $\|u\|_{L^{\infty}} \leq\|u\|_{H^{1}}, g(u) \in C^{\infty}(R)$, and $g(0)=0$, we deduce that

$$
\begin{aligned}
|g(u(x))| & \leq \sup _{|v| \leq\|u\|_{L^{\infty}}}\left|g^{\prime}(v)\right||u(x)| \leq c\left(\|u\|_{L^{\infty}}\right)|u(x)| \\
& \leq c\left(\|u\|_{H^{1}}\right)|u(x)| .
\end{aligned}
$$

Since $u, \varphi \in H^{1}$, Young's inequality ensures that $\Psi_{1}, \Psi_{2}, \Psi_{3} \in$ $H^{1}$.

Definition 1. For a solution of the Cauchy problem (6) on $\left[t_{1}, t_{2}\right]$, it means a Hölder continuous function $(u(t, x)$, $\eta(t, x))$ is defined on $\left[t_{1}, t_{2}\right] \times R$ with the following properties. At each fixed $t$ it has $(u(t, \cdot), \varphi(t, \cdot)) \in H^{1} \times\left(H^{1} \cap W^{1, \infty}\right)$. Moreover, the maps $u(t, \cdot)$ and $\varphi(t, \cdot)$ are Lipschitz continuous from $\left[t_{1}, t_{2}\right]$ to $L^{2}$, satisfying the initial condition and

$$
\begin{gathered}
u_{t}+u u_{x}=-\Psi_{1 x}, \quad t>0, x \in R, \\
\varphi_{t}+u \varphi_{x}=-\Psi_{2 x}-\Psi_{3}, \quad t>0, x \in R,
\end{gathered}
$$

where (9) is understood as equalities between functions in $L^{2}$.

For smooth solutions, we have the conservation law

$$
\begin{aligned}
E(t) & =\int_{R}\left(u^{2}+u_{x}^{2}+\varphi^{2}+\varphi_{x}^{2}\right) d x \\
& =\int_{R}\left(u_{0}^{2}+u_{0 x}^{2}+\varphi_{0}^{2}+\varphi_{0 x}^{2}\right) d x \\
& =E(0)=E_{0} .
\end{aligned}
$$

Indeed, differentiating the first equation of (6) with respect to $x$ and using the identity $\partial_{x}^{2} p * f=p * f-f$, we have

$$
u_{t x}+u u_{x x}+u_{x}^{2}=f-p * f
$$

where $f=u^{2}+(1 / 2) u_{x}^{2}+g(u)+(1 / 2) \varphi^{2}-(1 / 2) \varphi_{x}^{2}$. Using problem (6) and the above equality, we have

$$
\begin{aligned}
\int_{R}\left(u u_{t}+u_{x} u_{t x}\right) d x & \\
=\int_{R}\{ & -u^{2} u_{x}-\partial_{x} p *\left[u^{2}+\frac{1}{2} u_{x}^{2}+g(u)+\frac{1}{2} \varphi^{2}+\frac{1}{2} \varphi_{x}^{2}\right] u \\
& -u_{x}^{3}-u u_{x} u_{x x x}-\partial_{x}^{2} p \\
& \left.*\left[u^{2}+\frac{1}{2} u_{x}^{2}+g(u)+\frac{1}{2} \varphi^{2}+\frac{1}{2} \varphi_{x}^{2}\right] u_{x}\right\} \\
=\frac{1}{2} \int_{R} & \left(\varphi^{2} u_{x}-\varphi_{x}^{2} u_{x}\right) d x
\end{aligned}
$$

where $\int_{R} g(u) u_{x} d x=\left.G^{\prime}(u)\right|_{R}=0$.

In the same way, for the second equation in (6), we have

$$
\begin{aligned}
\int_{R}\left(\varphi \varphi_{t}+\varphi_{x} \varphi_{t x}\right) d x \\
\quad=\int_{R}\left(-u \varphi \varphi_{x}-\varphi_{x}\left(u \varphi_{x}\right)_{x}+u_{x} \varphi_{x}^{2}-u_{x} \varphi^{2}\right) d x
\end{aligned}
$$


Combining (12) with (13), we deduce that

$$
\begin{aligned}
& \frac{d}{d t} \int_{R}\left(u^{2}+u_{x}^{2}+\varphi^{2}+\varphi_{x}^{2}\right) d x \\
& =2 \int_{R}\left(u u_{t}+u_{x} u_{t x}+\varphi \varphi_{t}+\varphi_{x} \varphi_{t x}\right) d x \\
& =2 \int_{R}\left[\frac{1}{2} \varphi^{2} u_{x}-\frac{1}{2} \varphi_{x}^{2} u_{x}-u \varphi \varphi_{x}-\varphi_{x}\left(u \varphi_{x}\right)_{x}\right. \\
& \left.\quad+u_{x} \varphi_{x}^{2}-u_{x} \varphi^{2}\right] d x \\
& =\int_{R}\left(-u \varphi^{2}\right)_{x}-\left(u \varphi_{x}^{2}\right)_{x} d x=0 .
\end{aligned}
$$

Thus, (10) holds.

2.2. A New Set of Independent and Dependent Variables. Let $(\bar{u}, \bar{\varphi}) \in H^{1} \times\left(H^{1} \cap W^{1, \infty}\right)$ be the initial data. For an energy variable $\xi \in R$, let the nondecreasing map $\xi \mapsto \bar{y}(\xi)$ be defined by

$$
\xi=\int_{0}^{\bar{y}(\xi)}\left(1+\bar{u}_{x}^{2}\right) d x
$$

Assuming that the solution $(u, \eta)$ to $(6)$ remains Lipschitz continuous for $t \in[0, T]$, we derive an equivalent system of (6) by using the independent variables $(t, \xi)$. that

Let $\xi \mapsto y(\xi)$ be the characteristic starting at $\bar{y}(\xi)$ such

$$
\frac{\partial}{\partial t} y(t, \xi)=u(t, y(t, \xi)), \quad y(0, \xi)=\bar{y}(\xi)
$$

Moreover, we write

$$
\begin{array}{rr}
u(t, \xi) \doteq u(t, y(t, \xi)), & \varphi(t, \xi) \doteq \varphi(t, y(t, \xi)), \\
\varphi_{x}(t, \xi) \doteq \varphi_{x}(t, y(t, \xi)), & \\
\Psi_{i}(t, \xi) \doteq \Psi_{i}(t, y(t, \xi)), & \Psi_{i x}(t, \xi) \doteq \Psi_{i x}(t, y(t, \xi)), \\
& i=1,2,3 .
\end{array}
$$

The following further variables will be used: $v=v(t, \xi)$, $q=q(t, \xi)$, and $\psi=\psi(t, \xi)$, defined as

$$
v \doteq 2 \arctan u_{x}, \quad q \doteq\left(1+u_{x}^{2}\right) \cdot \frac{\partial y}{\partial \xi}, \quad \psi \doteq \varphi_{x}(t, \xi)
$$

Obviously, $v$ is defined up to multiples of $2 \pi$. Notice that (15) implies

$$
q(0, \xi)=1
$$

For future use, we write the identities

$$
\begin{gathered}
\frac{1}{1+u_{x}^{2}}=\cos ^{2} \frac{v}{2}, \quad \frac{u_{x}}{1+u_{x}^{2}}=\frac{1}{2} \sin v, \\
\frac{u_{x}^{2}}{1+u_{x}^{2}}=\sin ^{2} \frac{v}{2}, \\
\frac{\partial y}{\partial \xi}=\frac{q}{1+u_{x}^{2}}=\cos ^{2} \frac{v}{2} \cdot q .
\end{gathered}
$$

Using identity (21) yields

$$
y\left(t, \xi_{2}\right)-y\left(t, \xi_{1}\right)=\int_{\xi_{1}}^{\xi_{2}} \frac{\partial y}{\partial \xi} d \xi=\int_{\xi_{1}}^{\xi_{2}} \cos ^{2} \frac{v(t, \xi)}{2} q(t, \xi) d \xi .
$$

Furthermore, we have

$$
\begin{aligned}
\Psi_{1}(t, \xi)=\frac{1}{2} \int_{-\infty}^{\infty} & e^{-|y(t, \xi)-x|} \\
& \times\left(u^{2}+\frac{1}{2} u_{x}^{2}+g(u)+\frac{1}{2} \varphi^{2}-\frac{1}{2} \varphi_{x}^{2}\right) \\
& \times(t, x) d x
\end{aligned}
$$$$
\Psi_{1 x}(t, \xi)=\frac{1}{2}\left(\int_{y(t, \xi)}^{\infty}-\int_{-\infty}^{y(t, \xi)}\right) e^{-|y(t, \xi)-x|}
$$

$$
\begin{aligned}
& \times\left(u^{2}+\frac{1}{2} u_{x}^{2}+g(u)+\frac{1}{2} \varphi^{2}-\frac{1}{2} \varphi_{x}^{2}\right) \\
& \times(t, x) d x, \\
& \Psi_{2}(t, \xi)=\frac{1}{2} \int_{-\infty}^{\infty} e^{-|y(t, \xi)-x|} u_{x}(t, x) \varphi_{x}(t, x) d x, \\
& \Psi_{2 x}(t, \xi)=\frac{1}{2}\left(\int_{y(t, \xi)}^{\infty}-\int_{-\infty}^{y(t, \xi)}\right) e^{-|y(t, \xi)-x|} \\
& \times u_{x}(t, x) \varphi_{x}(t, x) d x,
\end{aligned}
$$

$\Psi_{3}(t, \xi)=\frac{1}{2} \int_{-\infty}^{\infty} e^{-|y(t, \xi)-x|} u_{x}(t, x) \varphi(t, x) d x$

$\Psi_{3 x}(t, \xi)=\frac{1}{2}\left(\int_{y(t, \xi)}^{\infty}-\int_{-\infty}^{y(t, \xi)}\right) e^{-|y(t, \xi)-x|} u_{x}(t, x) \varphi(t, x) d x$

In the above formulae, we use the change of variables $x=$ $y\left(t, \xi^{\prime}\right)$, the validity of which will be checked in Section 4 , and write the convolution as an integral over the variable $\xi^{\prime}$. Using 
identities (20)-(22), we obtain expressions for $\Psi_{i}$ and $\Psi_{i x}$ in terms of the new variable $\xi$, namely,

$$
\begin{aligned}
\Psi_{1}(\xi)=\frac{1}{2} \int_{-\infty}^{\infty} \exp \left\{-\left|\int_{\xi}^{\xi^{\prime}} \cos ^{2} \frac{v(s)}{2} q(s) d s\right|\right\} \\
\times\left[\left[u^{2}\left(\xi^{\prime}\right)+g\left(u\left(\xi^{\prime}\right)\right)\right] \cos ^{2} \frac{v\left(\xi^{\prime}\right)}{2}\right. \\
+\frac{1}{2} \sin ^{2} \frac{v\left(\xi^{\prime}\right)}{2}+\frac{1}{2} \varphi^{2}\left(\xi^{\prime}\right) \cos ^{2} \frac{v\left(\xi^{\prime}\right)}{2} \\
\left.-\frac{1}{2} \psi^{2}\left(\xi^{\prime}\right) \cos ^{2} \frac{v\left(\xi^{\prime}\right)}{2}\right] q\left(\xi^{\prime}\right) d \xi^{\prime},
\end{aligned}
$$

$$
\begin{aligned}
\Psi_{1 x}(\xi)=\frac{1}{2}\left(\int_{\xi}^{\infty}\right. & \left.-\int_{-\infty}^{\xi}\right) \exp \left\{-\left|\int_{\xi}^{\xi^{\prime}} \cos ^{2} \frac{v(s)}{2} q(s) d s\right|\right\} \\
\times[ & {\left[u^{2}\left(\xi^{\prime}\right)+g\left(u\left(\xi^{\prime}\right)\right)\right] \cos ^{2} \frac{v\left(\xi^{\prime}\right)}{2} } \\
& +\frac{1}{2} \sin ^{2} \frac{v\left(\xi^{\prime}\right)}{2}+\frac{1}{2} \varphi^{2}\left(\xi^{\prime}\right) \cos ^{2} \frac{v\left(\xi^{\prime}\right)}{2} \\
& \left.-\frac{1}{2} \psi^{2}\left(\xi^{\prime}\right) \cos ^{2} \frac{v\left(\xi^{\prime}\right)}{2}\right] q\left(\xi^{\prime}\right) d \xi^{\prime},
\end{aligned}
$$

$$
\begin{gathered}
\Psi_{2}(\xi)=\frac{1}{2} \int_{-\infty}^{\infty} \exp \left\{-\left|\int_{\xi}^{\xi^{\prime}} \cos ^{2} \frac{v(s)}{2} q(s) d s\right|\right\} \\
\times \frac{1}{2} \psi\left(\xi^{\prime}\right) \sin v\left(\xi^{\prime}\right) q\left(\xi^{\prime}\right) d \xi^{\prime},
\end{gathered}
$$

$$
\begin{gathered}
\Psi_{2 x}(\xi)=\frac{1}{2}\left(\int_{\xi}^{\infty}-\int_{-\infty}^{\xi}\right) \exp \left\{-\left|\int_{\xi}^{\xi^{\prime}} \cos ^{2} \frac{v(s)}{2} q(s) d s\right|\right\} \\
\cdot \frac{1}{2} \psi\left(\xi^{\prime}\right) \sin v\left(\xi^{\prime}\right) q\left(\xi^{\prime}\right) d \xi^{\prime},
\end{gathered}
$$$$
\Psi_{3}(\xi)=\frac{1}{2} \int_{-\infty}^{\infty} \exp \left\{-\left|\int_{\xi}^{\xi^{\prime}} \cos ^{2} \frac{v(s)}{2} q(s) d s\right|\right\}
$$$$
\times \frac{1}{2} \varphi\left(\xi^{\prime}\right) \sin v\left(\xi^{\prime}\right) q\left(\xi^{\prime}\right) d \xi^{\prime},
$$

$$
\begin{gathered}
\Psi_{3 x}(\xi)=\frac{1}{2}\left(\int_{\xi}^{\infty}-\int_{-\infty}^{\xi}\right) \exp \left\{-\left|\int_{\xi}^{\xi^{\prime}} \cos ^{2} \frac{v(s)}{2} q(s) d s\right|\right\} \\
\cdot \frac{1}{2} \varphi\left(\xi^{\prime}\right) \sin v\left(\xi^{\prime}\right) q\left(\xi^{\prime}\right) d \xi^{\prime} .
\end{gathered}
$$

From (6) and (16), the evolution equations for $u$ and $\eta$ in the new variables $(t, \xi)$ take the form

$$
\begin{gathered}
\frac{\partial}{\partial t} u(t, \xi)=u_{t}+u u_{x}=-\Psi_{1 x}(t, \xi), \\
\frac{\partial}{\partial t} \varphi(t, \xi)=\varphi_{t}+u \varphi_{x}=-\Psi_{2 x}(t, \xi)-\Psi_{3}(t, \xi),
\end{gathered}
$$

where $\Psi_{i}$ and $\Psi_{i x}(i=1,2,3)$ are given in (24)-(29).

Next, to derive an evolution equation for the variable $q$, we observe from (16) that

$$
\int_{\xi_{1}}^{\xi_{2}} q(t, \xi) d \xi=\int_{y\left(t, \xi_{1}\right)}^{y\left(t, \xi_{2}\right)}\left(1+u_{x}^{2}(t, x)\right) d x
$$

holds. We have the equality

$$
\begin{aligned}
\frac{d}{d t} \int_{\xi_{1}}^{\xi_{2}} q(t, \xi) d \xi & =\int_{y\left(t, \xi_{1}\right)}^{y\left(t, \xi_{2}\right)}\left\{\left(1+u_{x}^{2}\right)_{t}+\left[u\left(1+u_{x}^{2}\right)\right]_{x}\right\} d x \\
& =\int_{y\left(t, \xi_{1}\right)}^{y\left(t, \xi_{2}\right)}\left[2 u_{x} u_{x t}+u_{x}+\left(u u_{x}^{2}\right)_{x}\right] d x \\
& =\int_{y\left(t, \xi_{1}\right)}^{y\left(t, \xi_{2}\right)}\left[2 u^{2}+2 g(u)+\varphi^{2}-\varphi_{x}^{2}\right. \\
\left.-2 \Psi_{1}+1\right] u_{x} d x . &
\end{aligned}
$$

Therefore, it has

$$
\frac{\partial}{\partial t} q(t, \xi)=\left(u^{2}+g(u)+\frac{1}{2} \varphi^{2}-\frac{1}{2} \psi^{2}-\Psi_{1}+\frac{1}{2}\right) \sin v \cdot q \text {. }
$$

Applying (6), (9), and (16)-(18) yields

$$
\begin{gathered}
\frac{\partial}{\partial t} v(t, \xi)=\left(2 u^{2}+2 g(u)+\varphi^{2}-\psi^{2}-2 \Psi_{1}\right) \cos ^{2} \frac{\nu}{2}-\sin ^{2} \frac{\nu}{2}, \\
\frac{\partial}{\partial t} \psi(t, \xi)=-\Psi_{2}-\Psi_{3 x} .
\end{gathered}
$$

\section{Global Solutions of the Semilinear System}

Let initial data $(\bar{u}, \bar{\varphi}) \in H^{1} \times\left(H^{1} \cap W^{1, \infty}\right)$ be given. From Section 2, we rewrite the corresponding Cauchy problem (6) for the variables $(u, \varphi, \psi, v, q)$ in the form

$$
\begin{gathered}
\frac{\partial u}{\partial t}=-\Psi_{1 x}, \\
\frac{\partial \varphi}{\partial t}=-\Psi_{2 x}-\Psi_{3}, \\
\frac{\partial \psi}{\partial t}=-\Psi_{2}-\Psi_{3 x}, \\
\frac{\partial v}{\partial t}=\left(2 u^{2}+2 g(u)+\varphi^{2}-\psi^{2}-2 \Psi_{1}\right) \cos ^{2} \frac{v}{2}-\sin ^{2} \frac{v}{2}, \\
\frac{\partial q}{\partial t}=\left(u^{2}+g(u)+\frac{1}{2} \varphi^{2}-\frac{1}{2} \psi^{2}-\Psi_{1}+\frac{1}{2}\right) \sin v \cdot q,
\end{gathered}
$$


with initial data

$$
\begin{gathered}
u(0, \xi)=\bar{u}(\bar{y}(\xi)), \\
\varphi(0, \xi)=\bar{\varphi}(\bar{y}(\xi)), \\
\psi(0, \xi)=\bar{\varphi}_{x}(\bar{y}(\xi)), \\
v(0, \xi)=2 \arctan \bar{u}_{x}(\bar{y}(\xi)), \\
q(0, \xi)=1 .
\end{gathered}
$$

We regard (35) as an ordinary differential equation in the Banach space:

$$
X \doteq H^{1} \times H^{1} \times\left(L^{2} \cap L^{\infty}\right) \times\left(L^{2} \cap L^{\infty}\right) \times L^{\infty},
$$

with the norm

$$
\begin{aligned}
\|(u, \varphi, \psi, v, q)\|_{X} \doteq & \|u\|_{H^{1}}+\|\varphi\|_{H^{1}}+\|\psi\|_{L^{2}} \\
& +\|\psi\|_{L^{\infty}}+\|v\|_{L^{2}}+\|v\|_{L^{\infty}}+\|q\|_{L^{\infty}}
\end{aligned}
$$

Then the solution of the Cauchy problem means a fixed point of the integral transformation:

$$
T(u, \varphi, \psi, v, q)=(\tilde{u}, \tilde{\varphi}, \tilde{\psi}, \widetilde{v}, \tilde{q})
$$

where

$$
\begin{gathered}
\tilde{u}(t, \xi)=\bar{u}(\bar{y}(\xi))-\int_{0}^{t} \Psi_{1 x} d t \\
\tilde{\varphi}(t, \xi)=\bar{\varphi}(\bar{y}(\xi))-\int_{0}^{t}\left(\Psi_{2 x}+\Psi_{3}\right) d t \\
\tilde{\psi}(t, \xi)=\bar{\varphi}_{x}(\bar{y}(\xi))-\int_{0}^{t}\left(\Psi_{2}+\Psi_{3 x}\right) d t, \\
\tilde{v}(t, \xi)=2 \arctan \bar{u}_{x}(\bar{y}(\xi)) \\
+\int_{0}^{t}\left[\left(2 u^{2}+2 g(u)+\varphi^{2}-\psi^{2}-2 \Psi_{1}\right)\right. \\
\left.\times \cos ^{2} \frac{v}{2}-\sin ^{2} \frac{v}{2}\right] d t, \\
\tilde{q}(t, \xi)=1+\int_{0}^{t}\left(u^{2}+g(u)+\frac{1}{2} \varphi^{2}-\frac{1}{2} \psi^{2}\right. \\
\left.-\Psi_{1}+\frac{1}{2}\right) \sin v \cdot q d t .
\end{gathered}
$$

Proving the local Lipschitz continuity of the right-hand side of (35), the local existence of solution follows from the standard theorem for ordinary differential equations in Banach spaces. Then, we show the conservation of energy property expressed by (10). Moreover, we prove that this local solution can be extended globally in time.

Theorem 2. If $(\bar{u}, \bar{\varphi}) \in H^{1} \times\left(H^{1} \cap W^{1, \infty}\right)$, then the Cauchy problem (35)-(36) has a unique solution for all $t \geq 0$ in the sense of Definition 1.
Proof.

Step 1 (local solution). In order to establish the local existence of solution, it suffices to show that the operator determined by the right-hand side of (35) mapping $(u, \varphi, \psi, v, q)$ to

$$
\begin{aligned}
& \left(-\Psi_{1 x},-\Psi_{2 x}-\Psi_{3},-\Psi_{2}-\Psi_{3 x},\right. \\
& \left(2 u^{2}+2 g(u)+\varphi^{2}-\psi^{2}-2 \Psi_{1}\right) \\
& \times \cos ^{2} \frac{v}{2}-\sin ^{2} \frac{v}{2}, \\
& \left.\left(u^{2}+g(u)+\frac{1}{2} \varphi^{2}-\frac{1}{2} \psi^{2}-\Psi_{1}+\frac{1}{2}\right) \sin v \cdot q\right)
\end{aligned}
$$

is Lipschitz continuous on every bounded domain $\Omega \subset X$ in the form of

$$
\begin{gathered}
\Omega=\left\{(u, \varphi, \psi, v, q):\|u\|_{H^{1}} \leq \alpha,\|\varphi\|_{H^{1}} \leq \beta,\right. \\
\|\psi\|_{L^{2}} \leq m,\|\psi\|_{L^{\infty}} \leq \gamma, \\
\left.\|v\|_{L^{2}} \leq n,\|v\|_{L^{\infty}} \leq \frac{3 \pi}{2}, q(x) \in\left[q^{-}, q^{+}\right]\right\},
\end{gathered}
$$

for any constants $\alpha, \beta, m, n, \gamma, q^{-}, q^{+}>0$.

Applying Sobolev's inequality $\|u\|_{L^{\infty}} \leq\|u\|_{H^{1}},\|\varphi\|_{L^{\infty}} \leq$ $\|\varphi\|_{H^{1}}$, we know that the maps

$$
\begin{gathered}
u^{2}, \quad g(u), \quad g(u) \cos v, \quad u^{2} \cos ^{2} \frac{v}{2}, \quad \varphi^{2}, \quad \varphi^{2} \cos ^{2} \frac{v}{2}, \\
\sin v, \quad \sin ^{2} \frac{v}{2}, \quad \sin v \cdot q, \quad \varphi^{2}, \quad \varphi^{2} \cos ^{2} \frac{v}{2}
\end{gathered}
$$

are all Lipschitz continuous from $\Omega \mapsto L^{2} \cap L^{\infty}$. Now, we only need to prove the Lipschitz continuity of the maps

$$
(u, \varphi, \psi, v, q) \longmapsto \Psi_{i}, \quad(u, \varphi, \psi, v, q) \longmapsto \Psi_{i x}, \quad i=1,2,3,
$$

defined in (24)-(29) from $\Omega$ to $H^{1}$. This will also imply the Lipschitz continuity of these maps from $\Omega$ to $L^{2} \cap L^{\infty}$. To get this goal, we first observe that, as long as $|v| \leq 3 \pi / 2$, it holds that

$$
\begin{aligned}
& \sin ^{2} \frac{v}{2} \leq \frac{v^{2}}{4} \leq \frac{9 \pi^{2}}{8} \sin ^{2} \frac{v}{2}, \\
& \text { measure }\left\{\xi \in R ;\left|\frac{v(\xi)}{2}\right| \geq \frac{\pi}{4}\right\} \\
& \leq \text { measure }\left\{\xi \in R ; \sin ^{2} \frac{v(\xi)}{2} \geq \frac{1}{18}\right\} \\
& \leq 18 \int_{\left\{\xi \in R ; \sin ^{2}(v(\xi) / 2) \geq 1 / 18\right\}} \sin ^{2} \frac{v(\xi)}{2} d \xi \\
& \leq \frac{9}{2} n^{2} .
\end{aligned}
$$


Therefore, for any $\xi_{1}<\xi_{2}$, it holds that

$$
\begin{aligned}
\int_{\xi_{1}}^{\xi_{2}} \cos ^{2} \frac{v(\xi)}{2} q\left(\xi^{\prime}\right) d \xi & \geq \int_{\left\{\xi \in\left[\xi_{1}, \xi_{2}\right]:|v(\xi) / 2| \leq \pi / 4\right\}} \frac{q^{-}}{2} d \xi \\
& \geq q^{-}\left(\frac{\xi_{1}-\xi_{2}}{2}-\frac{9}{4} n^{2}\right) .
\end{aligned}
$$

Introducing the exponentially decaying function

$$
F(\zeta) \doteq \min \left\{1, \exp \left(\frac{9}{4} n^{2} q^{-}-\frac{|\zeta|}{2} q^{-}\right)\right\}
$$

we show that

$$
\|F\|_{L^{1}}=\left(\int_{|\zeta| \leq(9 / 2) n^{2}}+\int_{|\zeta| \geq(9 / 2) n^{2}}\right) F(\zeta) d \zeta=9 n^{2}+\frac{4}{q^{-}}
$$

Next, we begin to show $\Psi_{i}, \Psi_{i x} \in H^{1}, i=1,2,3$. We only give the estimates for $\Psi_{1}$, since the estimates for others are similar. It follows from definition (24) that

$$
\begin{aligned}
\left|\Psi_{1}(\xi)\right| \leq \frac{q^{+}}{2} \mid F^{*} & \left(\left(u^{2}+g(u)\right) \cos ^{2} \frac{v}{2}\right. \\
& +\frac{1}{2} \sin ^{2} \frac{v}{2}+\frac{1}{2} \varphi^{2} \cos ^{2} \frac{v}{2} \\
& \left.-\frac{1}{2} \psi^{2} \cos ^{2} \frac{v}{2}\right)(\xi) \mid .
\end{aligned}
$$

Using standard properties of convolutions, we obtain

$$
\begin{aligned}
\left\|\Psi_{1}(\xi)\right\|_{L^{2}} \leq & \frac{q^{+}}{2}\|F\|_{L^{1}} \\
& \cdot\left(\left\|u^{2}+g(u)\right\|_{L^{2}}+\frac{1}{8}\left\|v^{2}\right\|_{L^{2}}+\frac{1}{2}\left\|\varphi^{2}\right\|_{L^{2}}\right. \\
\left.\quad+\frac{1}{2}\left\|\psi^{2}\right\|_{L^{2}}\right) & \\
\leq & \frac{q^{+}}{2}\|F\|_{L^{1}}\left(C\|u\|_{L^{\infty}}\|u\|_{L^{2}}+\frac{1}{8}\|v\|_{L^{\infty}}\|v\|_{L^{2}}\right. \\
& \left.+\frac{1}{2}\|\varphi\|_{L^{\infty}}\|\varphi\|_{L^{2}}+\frac{1}{2}\|\psi\|_{L^{\infty}}\|\psi\|_{L^{2}}\right)
\end{aligned}
$$

$<\infty$ where we have used $H^{1} \subset L^{\infty}$ and (8). Differentiating (24), we get

$$
\begin{aligned}
\partial_{\xi} \Psi_{1}(\xi)= & \frac{1}{2} \int_{-\infty}^{\infty} \exp \left\{-\left|\int_{\xi}^{\xi^{\prime}} \cos ^{2} \frac{v(s)}{2} q(s) d s\right|\right\} \\
& \cdot \cos ^{2} \frac{v(\xi)}{2} q(\xi) \operatorname{sign}\left(\xi^{\prime}-\xi\right) \\
& \cdot\left[\left[u^{2}\left(\xi^{\prime}\right)+g\left(u\left(\xi^{\prime}\right)\right)\right] \cos ^{2} \frac{v\left(\xi^{\prime}\right)}{2}\right. \\
& +\frac{1}{2} \sin ^{2} \frac{v\left(\xi^{\prime}\right)}{2} \\
& +\frac{1}{2} \varphi^{2}\left(\xi^{\prime}\right) \cos ^{2} \frac{v\left(\xi^{\prime}\right)}{2} \\
& \left.-\frac{1}{2} \psi^{2}\left(\xi^{\prime}\right) \cos ^{2} \frac{v\left(\xi^{\prime}\right)}{2}\right] \\
& q\left(\xi^{\prime}\right) d \xi^{\prime}
\end{aligned}
$$

thus

$$
\begin{aligned}
&\left|\partial_{\xi} \Psi_{1}(\xi)\right| \leq \frac{1}{2}\left(q^{+}\right)^{2}\left|\cos ^{2} \frac{v}{2}\right| \\
& \cdot \mid F^{*}\left(\left[u^{2}+g(u)\right] \cos ^{2} \frac{v}{2}+\frac{1}{2} \sin ^{2} \frac{v}{2}\right. \\
&\left.+\frac{1}{2} \varphi^{2} \cos ^{2} \frac{v}{2}-\frac{1}{2} \psi^{2} \cos ^{2} \frac{v}{2}\right) \mid \\
& \leq \frac{1}{2}\left(q^{+}\right)^{2} \cdot \mid F^{*}\left[\left(u^{2}+g(u)+\frac{1}{2} \varphi^{2}-\frac{1}{2} \psi^{2}\right)\right. \\
&\left.\quad \times \cos ^{2} \frac{v}{2}+\frac{1}{2} \sin ^{2} \frac{v}{2}\right] \mid .
\end{aligned}
$$

Therefore, we have

$$
\left\|\partial_{\xi} \Psi_{1}\right\|_{L^{2}} \leq \frac{1}{2}\left(q^{+}\right)^{2}\|F\|_{L^{1}}
$$

$\cdot\left(C\|u\|_{L^{\infty}}\|u\|_{L^{2}}+\frac{1}{8}\|v\|_{L^{\infty}}\|v\|_{L^{2}}\right.$

$$
\begin{aligned}
& +\frac{1}{2}\|\varphi\|_{L^{\infty}}\|\varphi\|_{L^{2}} \\
& \left.+\frac{1}{2}\|\psi\|_{L^{\infty}}\|\psi\|_{L^{2}}\right)
\end{aligned}
$$

$<\infty$,

and $\Psi_{1} \in H^{1}(R)$.

To establish the Lipschitz continuity of (44), it suffices to show that their partial derivatives

$$
\begin{gathered}
\frac{\partial \Psi_{i}}{\partial u}, \frac{\partial \Psi_{i}}{\partial \varphi}, \frac{\partial \Psi_{i}}{\partial \psi}, \frac{\partial \Psi_{i}}{\partial v}, \frac{\partial \Psi_{i}}{\partial q}, \\
\frac{\partial \Psi_{i x}}{\partial u}, \frac{\partial \Psi_{i x}}{\partial \varphi}, \frac{\partial \Psi_{i x}}{\partial \psi}, \frac{\partial \Psi_{i x}}{\partial v}, \frac{\partial \Psi_{i x}}{\partial q}, \quad i=1,2,3
\end{gathered}
$$


are uniformly bounded as $(u, \varphi, \psi, v, q)$ ranges inside the domain $\Omega$. We observe that these derivatives are bounded operators from the appropriate space into $H^{1}$. For sake of illustration, we will work out the detailed estimate for $\partial \Psi_{1} / \partial u$. All other derivatives can be estimated by the same way.

For a given point $(u, \varphi, \psi, v, q) \in \Omega$, the partial derivative $\left(\partial \Psi_{1} / \partial u\right): H^{1} \rightarrow L^{2}$ is the linear operator defined by

$$
\begin{aligned}
& {\left[\frac{\partial \Psi_{1}(u, \varphi, \psi, v, q)}{\partial u} \cdot \tilde{u}\right](\xi)} \\
& =\frac{1}{2} \int_{-\infty}^{\infty} \exp \left\{-\left|\int_{\xi}^{\xi^{\prime}} \cos ^{2} \frac{v(s)}{2} q(s) d s\right|\right\} \\
& \cdot\left[2 u\left(\xi^{\prime}\right)+g^{\prime}\left(u\left(\xi^{\prime}\right)\right)\right] \cos ^{2} \frac{v\left(\xi^{\prime}\right)}{2} q\left(\xi^{\prime}\right) \\
& \cdot \tilde{u}\left(\xi^{\prime}\right) d \xi^{\prime} .
\end{aligned}
$$

Thus,

$$
\left\|\frac{\partial \Psi_{1}}{\partial u} \cdot \tilde{u}\right\|_{L^{2}} \leq \frac{q^{+}}{2}\left\|F *\left[2 u+g^{\prime}(u)\right]\right\|_{L^{2}} \cdot\|\widetilde{u}\|_{L^{\infty}} .
$$

By use of $\|\tilde{u}\|_{L^{\infty}} \leq\|\tilde{u}\|_{H^{1}}$ and $\left|g^{\prime}(u)\right| \leq C\left(\|u\|_{H^{1}}\right)|u(x)|$, the above operator norm satisfies

$$
\left\|\frac{\partial \Psi_{1}}{\partial u}\right\| \leq C q^{+}\left\|_{F}\right\|_{L^{1}}\|u\|_{L^{2}}<\infty .
$$

From (51), $\left(\partial\left(\partial_{\xi} \Psi_{1}\right) / \partial u\right): H^{1} \rightarrow L^{2}$ is the linear operator defined by

$$
\begin{aligned}
& {\left[\frac{\partial\left(\partial_{\xi} \Psi_{1}\right)(u, \varphi, \psi, v, q)}{\partial u} \cdot \tilde{u}\right](\xi)} \\
& =\frac{1}{2} \int_{-\infty}^{\infty} \exp \left\{-\left|\int_{\xi}^{\xi^{\prime}} \cos ^{2} \frac{v(s)}{2} q(s) d s\right|\right\} \\
& \cdot \cos ^{2} \frac{v(\xi)}{2} q(\xi) \operatorname{sign}\left(\xi^{\prime}-\xi\right) \\
& \cdot\left[2 u\left(\xi^{\prime}\right)+g^{\prime}\left(u\left(\xi^{\prime}\right)\right)\right] \\
& \cdot \cos ^{2} \frac{v\left(\xi^{\prime}\right)}{2} q\left(\xi^{\prime}\right) \cdot \tilde{u}\left(\xi^{\prime}\right) d \xi^{\prime},
\end{aligned}
$$

whose norm is bounded by

$$
\left\|\frac{\partial\left(\partial_{\xi} \Psi_{1}\right)}{\partial u}\right\| \leq C\left(q^{+}\right)^{2}\|F\|_{L^{1}}\|u\|_{L^{2}}<\infty
$$

Combining (57) and (59) yields the boundedness of $\partial \Psi_{1} / \partial u$ as a linear operator from $H^{1}$ to $H^{1}$. The bounds on the other partial derivatives in (54) are obtained by a similar way.

The local existence of a solution to the Cauchy problem (35)-(36) on some small time interval $[0, T]$ with $T>0$ now follows from the standard theory of ordinary differential equations in Banach space. Thus, we have showed that the right-hand side of (35) is Lipschitz continuity on a neighbourhood of the initial data in the space $X$.

Step 2 (extension to a global solution). To ensure that the local solution of (35) constructed above can be extended to a global solution defined for all $t \geq 0$, it suffices to show that the quantity

$$
\|u\|_{H^{1}}+\|\varphi\|_{H^{1}}+\|\psi\|_{L^{2}}+\|\psi\|_{L^{\infty}}+\|v\|_{L^{2}}+\|v\|_{L^{\infty}}+\|q\|_{L^{\infty}}
$$

remains uniformly bounded on any bounded time interval. The a priori bounds on (60) will actually follow from the conservation of the total energy (10). In the following, we rederive this energy conservation property in terms of the new variables $(u, \varphi, \psi, v, q)$ and $\xi$.

As long as the local solution of (35) is defined, we claim that

$$
\begin{gathered}
u_{\xi}=\frac{q}{2} \sin v \\
\varphi_{\xi}=\psi \cos ^{2} \frac{v}{2} q \\
\frac{d}{d t} \int_{R}\left(u^{2} \cos ^{2} \frac{v}{2}+\sin ^{2} \frac{v}{2}+\varphi^{2} \cos ^{2} \frac{v}{2}+\psi^{2} \cos ^{2} \frac{v}{2}\right) \cdot q d \xi=0 .
\end{gathered}
$$

Using (35) and (25) yields

$$
\begin{aligned}
u_{\xi t}= & {\left[\left(u^{2}+g(u)+\frac{1}{2} \varphi^{2}-\frac{1}{2} \psi^{2}\right) \cos ^{2} \frac{v}{2}+\frac{1}{2} \sin ^{2} \frac{v}{2}\right] } \\
& \cdot q-\cos ^{2} \frac{v}{2} q \cdot \Psi_{1} .
\end{aligned}
$$

From (35), we get

$$
\begin{aligned}
\left(\frac{q}{2} \sin v\right)_{t}= & \frac{q_{t}}{2} \sin v+\frac{q}{2} \cos v \cdot v_{t} \\
= & \frac{\sin v}{2}\left(u^{2}+g(u)+\frac{1}{2} \varphi^{2}-\frac{1}{2} \psi^{2}\right. \\
& \left.\quad-\Psi_{1}+\frac{1}{2}\right) \sin v \cdot q
\end{aligned}
$$

Thus, we have

$$
u_{\xi t}=\left(\frac{q}{2} \sin v\right)_{t}
$$


Moreover, at the initial time $t=0$, using (20) and (36), we get

$$
\frac{\partial u}{\partial \xi}=\frac{u_{x}}{1+u_{x}^{2}}=\frac{\sin v}{2}, \quad q \equiv 1 .
$$

Therefore, we obtain that (61) remains valid for all times $t \geq 0$ as long as the solution is defined. The (62) holds true by the same way.

To prove (63), we proceed as follows. From (35), we deduce that

$$
\begin{aligned}
\frac{d}{d t} \int_{R}( & \left.u^{2} \cos ^{2} \frac{v}{2}+\sin ^{2} \frac{v}{2}+\varphi^{2} \cos ^{2} \frac{v}{2}+\psi^{2} \cos ^{2} \frac{v}{2}\right) \cdot q d \xi \\
= & \int_{R}\left(\frac{3}{2} u^{2}+g(u)+\varphi^{2}-\Psi_{1}\right) \sin v \cdot q d \xi \\
& -2 \int_{R}\left[u \Psi_{1 x}+\varphi\left(\Psi_{2 x}+\Psi_{3}\right)+\psi\left(\Psi_{2}+\Psi_{3 x}\right)\right] \\
& \times \cos ^{2} \frac{v}{2} \cdot q d \xi
\end{aligned}
$$

On the other hand, from (24)-(29), we have

$$
\Psi_{i \xi}=\Psi_{i x} \cos ^{2} \frac{v}{2} \cdot q, \quad i=1,2,3 .
$$

Applying (61)-(62) and (68)-(69), we have

$$
\begin{gathered}
\frac{d}{d t} \int_{R}\left(u^{2} \cos ^{2} \frac{v}{2}+\sin ^{2} \frac{v}{2}+\varphi^{2} \cos ^{2} \frac{v}{2}+\psi^{2} \cos ^{2} \frac{v}{2}\right) \cdot q d \xi \\
=\int_{R}\left(3 u^{2}+2 g(u)+2 \varphi^{2}-2 \Psi_{1}\right) u_{\xi} d \xi \\
\quad-2 \int_{R}\left(u \Psi_{1 \xi}+\varphi \Psi_{2 \xi}+\varphi \Psi_{3} \cos ^{2} \frac{v}{2} \cdot q\right. \\
\left.\quad+\varphi_{\xi} \Psi_{2}+\varphi_{\xi} \Psi_{3 \xi}\right) d \xi \\
=2 \int_{R}\left[\varphi^{2} u_{\xi}-\varphi \Psi_{3} \cos ^{2} \frac{v}{2} \cdot q\right. \\
\left.=0, \quad+\varphi\left(\Psi_{3} \cos ^{2} \frac{v}{2} \cdot q-\varphi \sin v \cdot \frac{v}{2}\right)\right]
\end{gathered}
$$

which implies (63).

We can now rewrite the total energy (10) in terms of the new variables. According to (70), we have that the energy remains constant in time,

$$
\begin{aligned}
E(t)=\int_{R}\left(u^{2}(t, \xi) \cos ^{2} \frac{v(t, \xi)}{2}+\sin ^{2} \frac{v(t, \xi)}{2}\right. \\
+\varphi^{2}(t, \xi) \cos ^{2} \frac{v(t, \xi)}{2} \\
\left.+\psi^{2}(t, \xi) \cos ^{2} \frac{v(t, \xi)}{2}\right) \cdot q(t, \xi) d \xi \\
=E(0)=E_{0},
\end{aligned}
$$

along any solution of (35)-(36).
From (61) and (71), we have

$$
\begin{aligned}
& \sup _{\xi \in R}\left|u^{2}(t, \xi)\right| \leq 2 \int_{R}\left|u u_{\xi}\right| d \xi \\
& \leq 2 \int_{R}|u|\left|\sin \frac{v}{2} \cos \frac{v}{2}\right| q d \xi \leq E_{0} \\
& \sup _{\xi \in R}\left|\varphi^{2}(t, \xi)\right| \leq 2 \int_{R}\left|\varphi \varphi_{\xi}\right| d \xi \leq 2 \int_{R}\left|\varphi \psi \cos ^{2} \frac{v}{2}\right| q d \xi \leq E_{0} .
\end{aligned}
$$

Applying (8) and (72), we get $|g(u)| \leq C E_{0}$. Moreover, it holds that

$$
\begin{aligned}
& \left\|\Psi_{1}\right\|_{L^{\infty}},\left\|\Psi_{1 x}\right\|_{L^{\infty}} \\
& \leq\left\|\frac{1}{2} e^{|\xi|}\right\|_{L^{\infty}} \\
& \quad \cdot\left\|\left[\left(u^{2}+g(u)+\varphi^{2}+\psi^{2}\right) \cos ^{2} \frac{v}{2}+\sin ^{2} \frac{v}{2}\right] \cdot q\right\|_{L^{1}} \\
& \leq C E_{0} .
\end{aligned}
$$

Notice that

$$
\begin{aligned}
& \varphi\left(\xi^{\prime}\right) \sin v\left(\xi^{\prime}\right) \leq \varphi(\xi)^{2} \cos ^{2} \frac{v\left(\xi^{\prime}\right)}{2}+\sin ^{2} \frac{v\left(\xi^{\prime}\right)}{2} \\
& \psi\left(\xi^{\prime}\right) \sin v\left(\xi^{\prime}\right) \leq \psi(\xi)^{2} \cos ^{2} \frac{v\left(\xi^{\prime}\right)}{2}+\sin ^{2} \frac{v\left(\xi^{\prime}\right)}{2}
\end{aligned}
$$

Similar calculations yield

$$
\begin{array}{ll}
\left\|\Psi_{2}\right\|_{L^{\infty}}, & \left\|\Psi_{2 x}\right\|_{L^{\infty}} \leq C E_{0}, \\
\left\|\Psi_{3}\right\|_{L^{\infty}}, & \left\|\Psi_{3 x}\right\|_{L^{\infty}} \leq C E_{0} .
\end{array}
$$

Noting (35) and (76), we deduce that, as long as the solution is defined,

$$
\begin{gathered}
\left|\psi_{t}\right| \leq E_{0}, \\
\|\psi\|_{L^{\infty}} \leq E_{0} t+\left\|\bar{\varphi}_{x}\right\|_{L^{\infty}} .
\end{gathered}
$$

Thus, we have

$$
\left|q_{t}\right| \leq C E_{0}\|q\|_{L^{\infty}}, \quad C \geq 0 .
$$

Since $q(0, \xi)=1$, using Gronwall's inequality, it holds that

$$
e^{-C E_{0} t} \leq\|q(t)\|_{L^{\infty}} \leq e^{C E_{0} t}
$$

From (35), it derives that

$$
\|v(t)\|_{L^{\infty}} \leq e^{M t}, \quad M=M\left(E_{0},\|\bar{\varphi}\|_{W^{1, \infty}}\right) .
$$


Moreover, (35) implies

$$
\begin{gathered}
\left|\frac{d}{d t}\left(\int_{R} u^{2}(t, \xi) d \xi\right)\right| \leq 2\|u(t)\|_{L^{\infty}}\left\|\Psi_{1 x}(t)\right\|_{L^{1}}, \\
\left|\frac{d}{d t}\left(\int_{R} u_{\xi}^{2}(t, \xi) d \xi\right)\right| \leq 2\left\|u_{\xi}(t)\right\|_{L^{\infty}}\left\|\partial_{\xi} \Psi_{1 x}(t)\right\|_{L^{1}}, \\
\left|\frac{d}{d t}\left(\int_{R} \varphi^{2}(t, \xi) d \xi\right)\right| \leq 2\|\varphi(t)\|_{L^{\infty}}\left(\left\|\Psi_{2 x}(t)\right\|_{L^{1}}+\left\|\Psi_{3}\right\|_{L^{\infty}}\right), \\
\left|\frac{d}{d t}\left(\int_{R} \varphi_{\xi}^{2}(t, \xi) d \xi\right)\right| \\
\leq 2\left\|\varphi_{\xi}(t)\right\|_{L^{\infty}}\left(\left\|\partial_{\xi} \Psi_{2 x}(t)\right\|_{L^{1}}+\left\|\partial_{\xi} \Psi_{3}\right\|_{L^{\infty}}\right) .
\end{gathered}
$$

From (61)-(63) and (72)-(73), we get uniform bounds of $u, u_{\xi}, \varphi, \varphi_{\xi}$ on bounded time interval, respectively. The estimates on $\|u\|_{H^{1}}$ and $\|\varphi\|_{H^{1}}$ will follow from bounds on the $L^{1}$-norms of $\Psi_{i x}(t)$ and $\partial_{\xi} \Psi_{i x}(t), i=1,2,3$. To get this goal, letting $r$ be the right-hand side of (79), we have $r^{-1} \leq$ $\|q(t)\|_{L^{\infty}} \leq r$. Indeed, from (8) and (35), we have

$$
\left|\left[\left(g(u)+u^{2}\right) \cos ^{2} \frac{v}{2}+\frac{1}{2} \sin ^{2} \frac{v}{2}\right] q(t)\right| \leq C E_{0} .
$$

It follows from (51) that

$$
\begin{aligned}
\left\|\partial_{\xi} \Psi_{1}(t)\right\|_{L^{1}} & \leq \frac{C}{2} E_{0} \int_{R} \exp \left\{-\left|\int_{\xi}^{\xi^{\prime}} r^{-1} \cos ^{2} \frac{v}{2} d s\right|\right\} d \xi \\
& \leq C E_{0}\|F\|_{L^{1}},
\end{aligned}
$$

where

$$
F(\zeta) \doteq \min \left\{1, \exp \left(9 E_{0} r^{-1}-r^{-1} \frac{|\zeta|}{2}\right)\right\} .
$$

We deduce

$$
\|F\|_{L^{1}} \leq 36 E_{0}+4 r .
$$

The estimate for $\left\|\partial_{\xi} \Psi_{1 x}\right\|_{L^{1}}$ is similar. Note that $\varphi \sin v \leq$ $\varphi^{2} \cos ^{2}(v / 2)+\sin ^{2}(v / 2), \psi \sin v \leq \psi^{2} \cos ^{2}(v / 2)+\sin ^{2}(v / 2) ;$ similar calculations show that the $L^{1}$-norms of $\Psi_{2 x}, \partial_{\xi} \Psi_{2 x}$, $\Psi_{3 x}, \partial_{\xi} \Psi_{3 x}$ are uniformly bounded. This proves the boundedness of $\|u\|_{H^{1}}$ and $\|\varphi\|_{H^{1}}$ for $t$ in bounded interval.

Finally, the equations in (35) imply that

$$
\frac{d}{d t}\|\psi\|_{L^{2}}^{2} \leq\|\psi\|_{L^{\infty}}\left(\left\|\Psi_{2}\right\|_{L^{1}}+\left\|\Psi_{3 x}\right\|_{L^{1}}\right) .
$$

Combining the bounds $\left\|\Psi_{2}\right\|_{L^{1}},\left\|\Psi_{3 x}\right\|_{L^{1}}$ with (77), we have that $\|\psi\|_{L^{\infty}}$ is uniformly bounded for $t$ in bounded interval. Thus,

$$
\begin{aligned}
& \frac{d}{d t}\|v\|_{L^{2}}^{2} \leq C\left(\|u\|_{L^{\infty}}\|u\|_{L^{2}}+\|\varphi\|_{L^{\infty}}\|\varphi\|_{L^{2}}\right. \\
&\left.+\|\psi\|_{L^{\infty}}\|\psi\|_{L^{2}}+\|v\|_{L^{\infty}}\|u\|_{L^{2}}\right) .
\end{aligned}
$$

By the previous bounds, it is clear that $\|v\|_{L^{2}}$ remains bounded on bounded time interval. This shows that the solution of (35) can be extended globally in time.
For future use, we record here a property of the solution. Namely, consider the set of times

$$
\mathfrak{R} \doteq\{t \geq 0: \text { measure }\{\xi \in R: v(t, \xi)=-\pi\}>0\} .
$$

Then, we claim

$$
\text { measure }(\mathfrak{R})=0 \text {. }
$$

Its validity will be proved in the next section.

\section{Solutions to the Original Equation}

We now show that the global solution of the system (35) yields a global conservative solution to system (6) in the original variables $(t, x)$.

Let us start with a global solution $(u, \varphi, \psi, v, q)$ to (35). Define

$$
y(t, \xi) \doteq \bar{y}(\xi)+\int_{0}^{t} u(\tau, \xi) d \tau
$$

For each fixed $\xi$, the function $t \mapsto y(t, \xi)$ provides a solution to the Cauchy problem:

$$
\frac{\partial}{\partial t} y(t, \xi)=u(t, \xi), \quad y(0, \xi)=\bar{y}(\xi)
$$

We claim that a solution of (6) can be obtained by setting

$$
u(t, x) \doteq u(t, \xi) ; \quad \varphi(t, x) \doteq \varphi(t, \xi), \quad \text { if } y(t, \xi)=x
$$

Theorem 3. Let $(u, \varphi, \psi, v, q)$ provide a global solution to (35)(36). Then the pair of functions $(u(t, x), \varphi(t, x))$ defined by (90)-(92) provide the global solution to the problem (6)-(7).

The solution $(u, \varphi)$ constructed in this way has the following properties. The energy is almost always conserved; namely,

$$
\|u(t)\|_{H^{1}}+\|\varphi(t)\|_{H^{1}}=\|\bar{u}(t)\|_{H^{1}}+\|\bar{\varphi}(t)\|_{H^{1}}, \quad \text { a.e. } t \geq 0 .
$$

Furthermore, consider the sequence of initial data $\left(\bar{u}_{n}, \bar{\varphi}_{n}\right)$, such that

$$
\begin{gathered}
\left\|\bar{u}_{n}-\bar{u}\right\|_{H^{1}} \longrightarrow 0, \quad\left\|\bar{\varphi}_{n}-\bar{\varphi}\right\|_{W^{1, \infty}} \longrightarrow 0, \\
\left\|\bar{\varphi}_{n}-\bar{\varphi}\right\|_{H^{1}} \longrightarrow 0 .
\end{gathered}
$$

Then the corresponding solutions $\left(u_{n}(t, x), \varphi_{n}(t, x)\right)$ converge to $(u(t, x), \varphi(t, x))$ uniformly for $(t, x)$ in any bounded sets.

Proof. Using the uniform bound $|u(t, \xi)| \leq E_{0}^{1 / 2}$ in (72) and combining with (90), we have the estimate

$$
\bar{y}(\xi)-E_{0}^{1 / 2} t \leq y(t, \xi) \leq \bar{y}(\xi)+E_{0}^{1 / 2} t, \quad t \geq 0 .
$$

Applying the definition of $\xi$ in (15), we obtain

$$
\lim _{\xi \rightarrow \pm \infty} \bar{y}(t, \xi)= \pm \infty .
$$


Therefore, the image of the continuous map $(t, \xi) \mapsto$ $(t, y(t, \xi))$ is the half-plane $R^{+} \times R$. Now we claim

$$
y_{\xi}=q \cos ^{2} \frac{v}{2}
$$

for all $t \geq 0$ and a.e. $\xi \in R$. Indeed, from (35) and (61) we have

$$
\frac{\partial}{\partial t}\left(q \cos ^{2} \frac{v}{2}\right)(t, \xi)=-q v_{t} \cos \frac{v}{2} \sin \frac{v}{2}+q_{t} \cos ^{2} \frac{v}{2}=u_{\xi}(t, \xi)
$$

On the other hand, (90) implies

$$
\frac{\partial}{\partial t} y_{\xi}(t, \xi)=u_{\xi}(t, \xi)
$$

Since the function $x \mapsto 2 \arctan \bar{u}_{x}(x)$ is measurable, identity (97) holds true for almost every $\xi \in R$ at $t=0$. By the above calculation it remains true for all $t \geq 0$.

Next, we prove the set $\mathfrak{R}$ that defined in (88) has measure zero. Indeed, if $v\left(t_{0}, \xi\right)=-\pi$, then $y_{\xi}\left(t_{0}, \xi\right)=$ $\cos ^{2}\left(v\left(t_{0}, \xi\right) / 2\right) q\left(t_{0}, \xi\right)=0$. Using (61), we get

$$
\frac{\partial}{\partial t} y_{\xi}\left(t_{0}, \xi\right)=u_{\xi}\left(t_{0}, \xi\right)=\frac{q}{2} \sin v\left(t_{0}, \xi\right)=0 .
$$

By (64), we have

$$
\frac{\partial^{2}}{\partial t^{2}} y_{\xi}\left(t_{0}, \xi\right)=u_{\xi t}\left(t_{0}, \xi\right)=\frac{1}{2} q\left(t_{0}, \xi\right)>0
$$

which implies that $t$ satisfying $y_{\xi}(t, \xi)=0$ is isolated. Thus, $t$ satisfying $v(t, \xi)=-\pi$ is also isolated. Since $v \in L^{2}$, it infers

$$
\text { measure }(\Re)=0 \text {. }
$$

From (97), we get $y(t, \xi)$ is nondeceasing. Moreover, if $\xi<$ $\xi^{\prime}$ and $y(t, \xi)=y\left(t, \xi^{\prime}\right)$, then

$$
\int_{\xi}^{\xi^{\prime}} y_{\xi}(t, s) d s=\int_{\xi}^{\xi^{\prime}} q(t, s) \cos ^{2} \frac{v(t, s)}{2} d s=0
$$

Hence, $\cos (v / 2) \equiv 0$ throughout the interval of integration. Therefore, from (61)-(62), we have

$$
\begin{gathered}
u\left(t, \xi^{\prime}\right)-u(t, \xi)=\int_{\xi}^{\xi^{\prime}} \frac{q(t, s)}{2} \sin v(t, s) d s=0, \\
\varphi\left(t, \xi^{\prime}\right)-\varphi(t, \xi)=\int_{\xi}^{\xi^{\prime}} \psi(t, s) q(t, s) \cos ^{2} \frac{v(t, s)}{2} d s=0,
\end{gathered}
$$

which proves that the map $(u(t, x), \varphi(t, x))$ in (92) is well defined for all $t \geq 0$ and $x \in R$.
If $x=y(t, \xi)$ holds, using the fact $\varphi_{\xi}=\psi q \cos ^{2}(v / 2)$, we have $\varphi_{x}(t, x)=\psi(t, \xi)$. This implies $\varphi_{x} \in L^{\infty}$. For every fixed $t$, we have

$$
\begin{gathered}
\int_{R}\left(u^{2}(t, x)+u_{x}^{2}(t, x)+\varphi^{2}(t, x)+\varphi_{x}^{2}(t, x)\right) d x \\
=\int_{\{\cos v>-1\}}\left(u^{2}(t, \xi) \cos ^{2} \frac{v(t, \xi)}{2}+\sin ^{2} \frac{v(t, \xi)}{2}\right. \\
+\varphi^{2}(t, \xi) \cos ^{2} \frac{v(t, \xi)}{2} \\
\left.+\psi^{2}(t, \xi) \cos ^{2} \frac{v(t, \xi)}{2}\right) \cdot q(t, \xi) d \xi \\
\leq E_{0} .
\end{gathered}
$$

Since the measure of $\mathfrak{R}$ is zero, the equality holds true for almost all $t$.

By Sobolev's inequality, this implies the uniform Hölder continuity with the exponent $1 / 2$ of $u$ and $\varphi$ as functions of $x$. By (35) and the bounds $\left\|\Psi_{1 x}\right\|_{L^{\infty}} \leq E_{0} / 2,\left\|\Psi_{2 x}\right\|_{L^{\infty}} \leq E_{0} / 2$, and $\left\|\Psi_{3}\right\|_{L^{\infty}} \leq E_{0} / 2$, we get that $(u(t, y(t)), \varphi(t, y(t)))$ is Hölder continuous with the exponent $1 / 2$. Indeed

$$
\begin{aligned}
|u(t, y)-u(s, x)| \leq & |u(t, y)-u(t, x)| \\
& +|u(t, x)-u(t, y(t, \xi))| \\
& +|u(t, y(t, \xi))-u(s, y(s, \xi))| \\
\leq & E_{0}^{1 / 2}|y-x|^{1 / 2} \\
& +E_{0}^{1 / 2}|y(t, \xi)-y(s, \xi)| \\
& +\int_{s}^{t}\left|\Psi_{1 x}(\tau, \xi)\right| d \tau \\
\leq & C\left(|y-x|^{1 / 2}+|t-s|^{1 / 2}+|t-s|\right),
\end{aligned}
$$

where we choose $\xi \in R$ such that the characteristic $t \mapsto y(t, \xi)$ passes through the point $(s, x)$. Notice that $u(t, x) \leq E_{0}^{1 / 2}$. This implies that $u(t, x)$ is uniform Hölder continuous with the exponent $1 / 2$. The same computation shows that $\varphi(t, x)$ is uniform Hölder continuous with the exponent $1 / 2$.

We now prove the Lipschitz continuity of $(u(t, x), \varphi(t, x))$ with values in $L^{2}$. Consider any interval $[\tau, \tau+h]$. For a given point $x$, we choose $\xi$ such that the characteristic $t \mapsto y(t, \xi)$ passes through the point $(\tau, x)$. By (35) and (72), it follows that

$$
\begin{aligned}
|u(\tau+h, x)-u(\tau, x)| \\
\leq|u(\tau+h, x)-u(\tau+h, y(\tau+h, \xi))| \\
\quad+|u(\tau+h, y(\tau+h, \xi))-u(\tau, x)| \\
\leq \sup _{|y-x| \leq E_{0}^{1 / 2} h}|u(\tau+h, y)-u(\tau+h, x)| \\
\quad+\int_{\tau}^{\tau+h}\left|\Psi_{1 x}(t, \xi)\right| d t .
\end{aligned}
$$


Integrating over the whole real line, in view of the boundedness of $\Psi_{1 x},(8),(79)$, and (105), we obtain

$$
\begin{aligned}
& \int_{R}|u(\tau+h, x)-u(\tau, x)|^{2} d x \\
& \leq 2 \int_{R}\left(\int_{x-E^{1 / 2} h}^{x+E^{1 / 2} h}\left|u_{x}(\tau+h, y)\right| d y\right)^{2} d x \\
& \quad+2 \int_{R}\left(\int_{\tau}^{\tau+h}\left|\Psi_{1 x}(t, \xi)\right| d t\right) q(\tau, \xi) \cos ^{2} \frac{v(\tau, \xi)}{2} d \xi \\
& \leq 2 \int_{R}\left(2 E_{0}^{1 / 2} h \int_{x-E_{0}^{1 / 2} h}^{x+E_{0}^{1 / 2} h}\left|u_{x}(\tau+h, y)\right|^{2} d y\right) d x \\
& \quad+2 \int_{R}\left(h \int_{\tau}^{\tau+h}\left|\Psi_{1 x}(t, \xi)\right|^{2} d t\right)\|q(\tau, \xi)\|_{L^{\infty}} d \xi \\
&=4 E_{0}^{1 / 2} h \int_{R}^{y+E_{0}^{1 / 2} h} \int_{y-E_{0}^{1 / 2} h}\left|u_{x}(\tau+h, y)\right|^{2} d x d y \\
& \quad+2 h\left\|_{q}(\tau)\right\|_{L^{\infty}} \int_{R} \int_{\tau}^{\tau+h}\left|\Psi_{1 x}(t, \xi)\right|^{2} d t d \xi \\
& \leq 8 E_{0} h^{2}\left\|u_{x}(\tau+h)\right\|_{L^{2}}^{2}+2 h\|q(\tau)\|_{L^{\infty}} \int_{\tau}^{\tau+h}\left\|\Psi_{1 x}(t)\right\|_{L^{2}}^{2} d t \\
& \leq C h^{2},
\end{aligned}
$$

where constant $C$ depends only on $T$. This clearly implies the Lipschitz continuity of the map $t \mapsto u(t)$ in terms of the $x$ variable. Similar calculation shows the Lipschitz continuity of $\varphi(t, x)$ as a map $t \mapsto L^{2}$.

Since $L^{2}$ is a reflexive space, the left-hand side of (6) is well defined for almost all $t \in R$. Note that we have proved that the right-hand side of (6) also lies in $L^{2}$ for almost all $t \in R$. To establish the equality between these two sides, we observe that

$$
\begin{gathered}
\frac{d}{d t} u(t, \xi)=-\Psi_{1 x}(t, \xi) \\
\frac{d}{d t} \varphi(t, \xi)=-\Psi_{2 x}(t, \xi)-\Psi_{3}(t, \xi) .
\end{gathered}
$$

On the other hand, recalling (88), for every $t \notin \mathfrak{R}$ the map $\xi \mapsto x(t, \xi)$ is one-to-one. Then, the change of variable formulae (24)-(29) yields

$$
\begin{aligned}
\Psi_{1 x}(t, \xi)= & \Psi_{1 x}(t, y(t, \xi)) \\
= & \frac{1}{2}\left(\int_{y(t, \xi)}^{\infty}-\int_{-\infty}^{y(t, \xi)}\right) e^{-|y(t, \xi)-x|} \\
& \times\left(u^{2}+\frac{1}{2} u_{x}^{2}+g(u)+\frac{1}{2} \varphi^{2}-\frac{1}{2} \varphi_{x}^{2}\right)(t, x) d x, \\
\Psi_{2 x}(t, \xi)= & \Psi_{2 x}(t, y(t, \xi)) \\
= & \frac{1}{2}\left(\int_{y(t, \xi)}^{\infty}-\int_{-\infty}^{y(t, \xi)}\right) e^{-|y(t, \xi)-x|} u_{x}(t, x) \varphi_{x}(t, x) d x,
\end{aligned}
$$

$$
\begin{aligned}
\Psi_{3}(t, \xi) & =\Psi_{3}(t, y(t, \xi)) \\
& =\frac{1}{2} \int_{-\infty}^{\infty} e^{-|y(t, \xi)-x|} u_{x}(t, x) \varphi(t, x) d x
\end{aligned}
$$

Hence, identity (6) is valid for almost all $t \geq 0$. This implies that $(u, \varphi)$ is a global solution of the generalized two-component Camassa-Holm system in the sense of Definition 1.

From (71) and (105), we obtain identity (93) for almost all $t \geq 0$.

Finally, let $\left(\bar{u}_{n}, \bar{\varphi}_{n}\right)$ be a sequence of initial data converging to $(\bar{u}, \bar{\varphi})$ in $H^{1} \times\left(H^{1} \cap W^{1, \infty}\right)$. In this case, from (35) and the boundedness of $u, \varphi, \psi$, we can infer $\left\|q_{n}(t, \xi)-q(t, \xi)\right\|_{L^{2}}<$ $\infty$. Recalling (15) and (36) at time $t=0$, we have

$$
\begin{gathered}
\sup _{\xi \in R}\left|y_{n}(0, \xi)-y(0, \xi)\right| \longrightarrow 0, \\
\left\|u_{n}(0, \xi)-u(0, \xi)\right\|_{H^{1}} \longrightarrow 0, \\
\left\|\varphi_{n}(0, \xi)-\varphi(0, \xi)\right\|_{H^{1}} \longrightarrow 0 \\
\left\|\psi_{n}(0, \xi)-\psi(0, \xi)\right\|_{L^{\infty}} \longrightarrow 0 \\
\left\|\psi_{n}(0, \xi)-\psi(0, \xi)\right\|_{L^{2}} \longrightarrow 0, \\
\left\|v_{n}(0, \xi)-v(0, \xi)\right\|_{L^{2}} \longrightarrow 0, \\
\left\|q_{n}(0, \xi)-q(0, \xi)\right\|_{L^{2}} \longrightarrow 0
\end{gathered}
$$

Now from (35) and the bounds of $u, \varphi, \psi, v, q$, we obtain

$$
\begin{aligned}
\frac{d}{d t}\left(\left\|u_{n}(t, \xi)-u(t, \xi)\right\|_{L^{\infty}}+\left\|\varphi_{n}(t, \xi)-\varphi(t, \xi)\right\|_{L^{\infty}}\right. \\
+\left\|\psi_{n}(t, \xi)-\psi(t, \xi)\right\|_{L^{\infty}} \\
+\left\|v_{n}(t, \xi)-v(t, \xi)\right\|_{L^{2}} \\
\left.\quad+\left\|q_{n}(t, \xi)-q(t, \xi)\right\|_{L^{2}}\right) \\
\leq C\left(\left\|u_{n}(t, \xi)-u(t, \xi)\right\|_{L^{\infty}}+\left\|\varphi_{n}(t, \xi)-\varphi(t, \xi)\right\|_{L^{\infty}}\right. \\
\quad+\left\|\psi_{n}(t, \xi)-\psi(t, \xi)\right\|_{L^{\infty}} \\
\left.\quad+\left\|v_{n}(t, \xi)-v(t, \xi)\right\|_{L^{2}}+\left\|q_{n}(t, \xi)-q(t, \xi)\right\|_{L^{2}}\right) .
\end{aligned}
$$

Thus, Gronwall's inequality implies that $u_{n}(t, \xi) \rightarrow$ $u(t, \xi), \varphi_{n}(t, \xi) \rightarrow \varphi(t, \xi)$, uniformly for $(t, \xi)$ in bounded sets. Returning to the original coordinates, it yields that

$$
\begin{gathered}
y_{n}(t, \xi) \longrightarrow y(t, \xi), \\
u_{n}(t, \xi) \longrightarrow u(t, \xi), \\
\varphi_{n}(t, \xi) \longrightarrow \varphi(t, \xi),
\end{gathered}
$$

uniformly on bounded sets since all functions $u, u_{n}, \varphi, \varphi_{n}$ are uniformly Hölder continuous.

\section{Conflict of Interests}

The authors declare that there is no conflict of interests regarding the publication of this paper. 


\section{Acknowledgment}

The authors acknowledge Teacher Ls Yong's help in modifying the English language of this paper.

\section{References}

[1] R. Camassa and D. D. Holm, "An integrable shallow water equation with peaked solitons," Physical Review Letters, vol. 71, no. 11, pp. 1661-1664, 1993.

[2] P. J. Olver and P. Rosenau, "Tri-Hamiltonian duality between solitons and solitary-wave solutions having compact support," Physical Review E, vol. 53, no. 2, pp. 1900-1906, 1996.

[3] A. Constantin and R. I. Ivanov, "On an integrable twocomponent Camassa-Holm shallow water system," Physics Letters A, vol. 372, no. 48, pp. 7129-7132, 2008.

[4] J. Escher, O. Lechtenfeld, and Z. Yin, "Well-posedness and blowup phenomena for the 2-component Camassa-Holm equation," Discrete and Continuous Dynamical Systems, vol. 19, no. 3, pp. 493-513, 2007.

[5] C. Guan and Z. Yin, "Global existence and blow-up phenomena for an integrable two-component Camassa-Holm shallow water system," Journal of Differential Equations, vol. 248, no. 8, pp. 2003-2014, 2010.

[6] G. Gui and Y. Liu, "On the global existence and wave-breaking criteria for the two-component Camassa-Holm system," Journal of Functional Analysis, vol. 258, no. 12, pp. 4251-4278, 2010.

[7] Q. Hu, "Global existence and blow-up phenomena for a weakly dissipative 2-component Camassa-Holm system," Applicable Analysis, vol. 92, no. 2, pp. 398-410, 2013.

[8] D. D. Holm, L. Ó’Náraigh, and C. Tronci, “Singular solutions of a modified two-component Camassa-Holm equation," Physical Review E, vol. 79, no. 1, Article ID 016601, 13 pages, 2009.

[9] W. Tan and Z. Yin, "Global conservative solutions of a modified two-component Camassa-Holm shallow water system," Journal of Differential Equations, vol. 251, no. 12, pp. 3558-3582, 2011.

[10] A. Constantin and J. Escher, "Well-posedness, global existence, and blowup phenomena for a periodic quasi-linear hyperbolic equation," Communications on Pure and Applied Mathematics, vol. 51, no. 5, pp. 475-504, 1998.

[11] A. Constantin, "Existence of permanent and breaking waves for a shallow water equation: a geometric approach," Annales de l'Institut Fourier, vol. 50, no. 2, pp. 321-362, 2000.

[12] S. Lai and Y. Wu, "Global solutions and blow-up phenomena to a shallow water equation," Journal of Differential Equations, vol. 249, no. 3, pp. 693-706, 2010.

[13] G. M. Coclite, H. Holden, and K. H. Karlsen, "Global weak solutions to a generalized hyperelastic-rod wave equation," SIAM Journal on Mathematical Analysis, vol. 37, no. 4, pp. 10441069, 2006.

[14] A. Constantin and L. Molinet, "Global weak solutions for a shallow water equation," Communications in Mathematical Physics, vol. 211, no. 1, pp. 45-61, 2000.

[15] S. Lai and Y. Wu, "The local well-posedness and existence of weak solutions for a generalized Camassa-Holm equation," Journal of Differential Equations, vol. 248, no. 8, pp. 2038-2063, 2010.

[16] S. Lai and Y. Wu, "Local well-posedness and weak solutions for a weakly dissipative Camassa-Holm equation," Scientia Sinica Mathematica, vol. 40, no. 9, pp. 901-920, 2010 (Chinese).
[17] A. Bressan and A. Constantin, "Global conservative solutions of the Camassa-Holm equation," Archive for Rational Mechanics and Analysis, vol. 183, no. 2, pp. 215-239, 2007.

[18] A. Bressan and A. Constantin, "Global dissipative solutions of the Camassa-Holm equation," Analysis and Applications, vol. 5, no. 1, pp. 1-27, 2007.

[19] H. Holden and X. Raynaud, "Global conservative solutions of the Camassa-Holm equation-a lagrangian point of view," Communications in Partial Differential Equations, vol. 32, no. 10-12, pp. 1511-1549, 2007.

[20] H. Holden and X. Raynaud, "Dissipative solutions for the camassa-holm equation," Discrete and Continuous Dynamical Systems A, vol. 24, no. 4, pp. 1047-1112, 2009.

[21] L. Tian and X. Song, "New peaked solitary wave solutions of the generalized Camassa-Holm equation," Chaos, Solitons and Fractals, vol. 19, no. 3, pp. 621-637, 2004.

[22] J. Shen and W. Xu, "Bifurcations of smooth and non-smooth travelling wave solutions in the generalized Camassa-Holm equation," Chaos, Solitons and Fractals, vol. 26, no. 4, pp. 1149$1162,2005$.

[23] O. G. Mustafa, "On the Cauchy problem for a generalized Camassa-Holm equation," Nonlinear Analysis, Theory, Methods \& Applications, vol. 64, no. 6, pp. 1382-1399, 2006.

[24] Z. Yin, "On the Cauchy problem for the generalized CamassaHolm equation," Nonlinear Analysis, Theory, Methods \& Applications, vol. 66, no. 2, pp. 460-471, 2007.

[25] S. Zhou and C. Mu, "Global conservative and dissipative solutions of the generalized Camassa-Holm equation," Discrete and Continuous Dynamical Systems A, vol. 33, no. 4, pp. 1713$1739,2013$. 


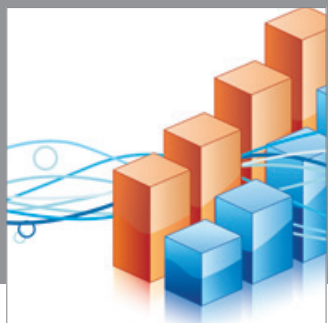

Advances in

Operations Research

mansans

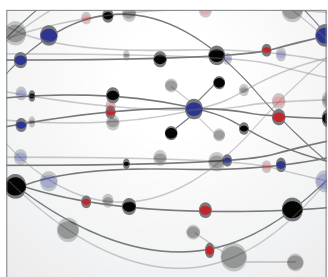

The Scientific World Journal
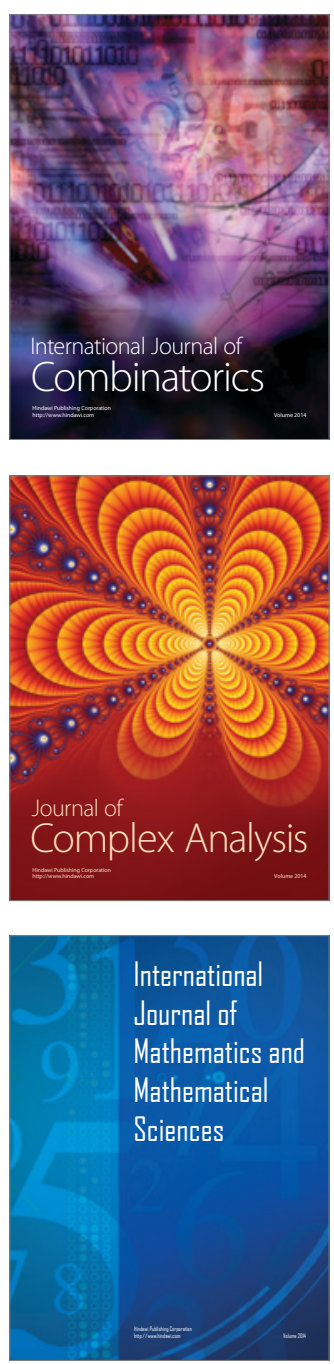
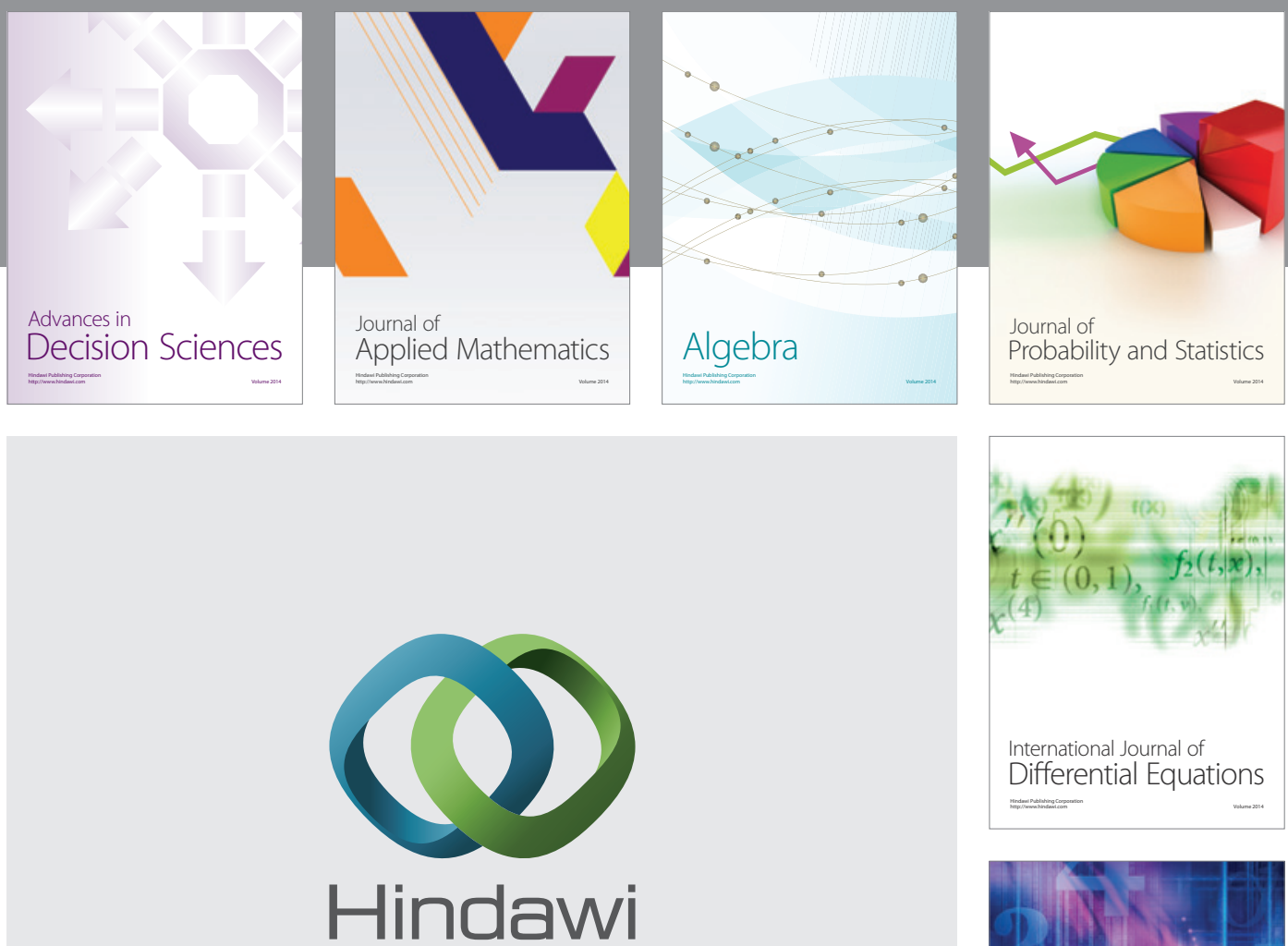

Submit your manuscripts at http://www.hindawi.com
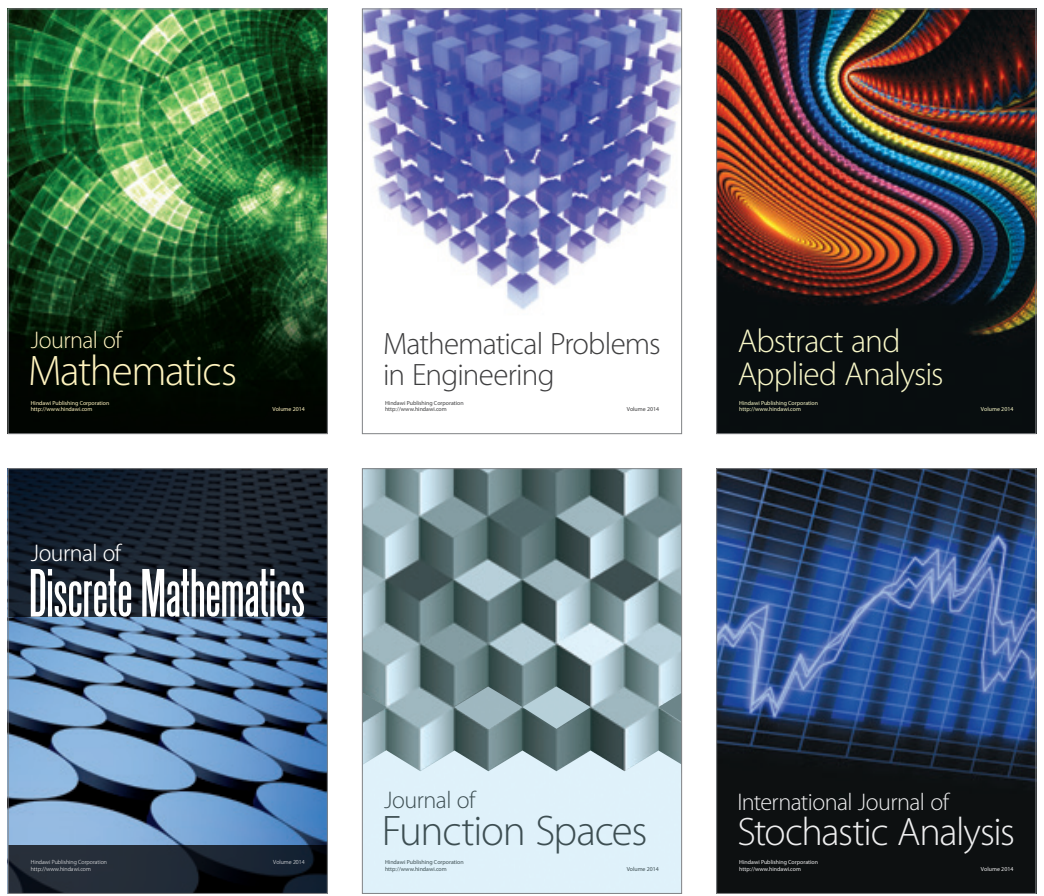

Journal of

Function Spaces

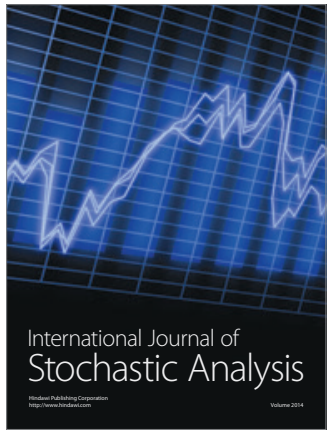

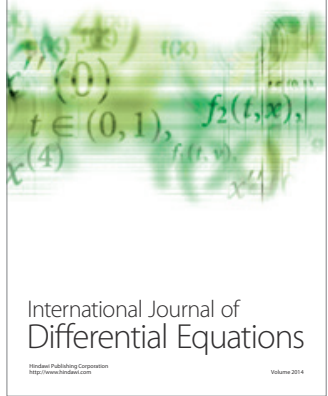
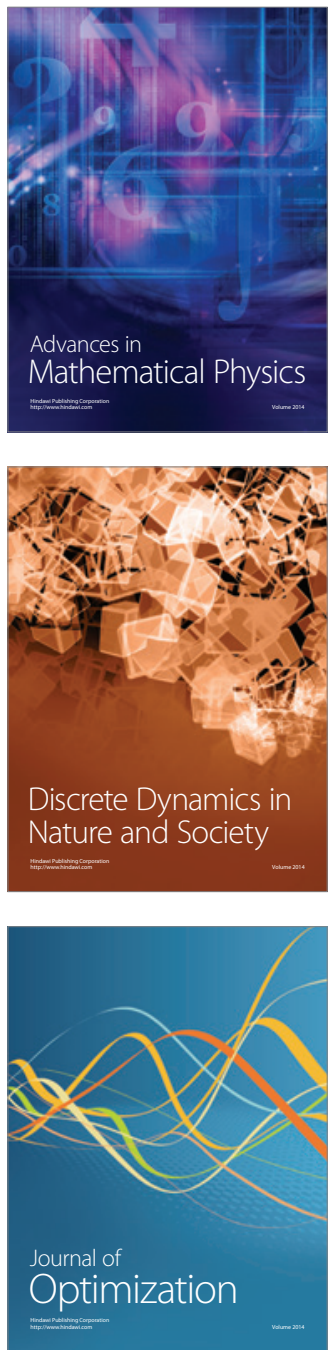